\title{
GRUNWALD-WANG THEOREM, AN EFFECTIVE VERSION
}

\author{
SONG WANG
}

\begin{abstract}
The main purpose of this note is to establish an effective version of the Grunwald-Wang Theorem, which asserts that given a family of local characters $\chi^{v}$ of $K_{v}^{*}$ of exponent $m$ where $v \in S$ for a finite set $S$ of primes of $K$, there exists a global character $\chi$ of the idele class group $C_{K}$ of exponent $m$ (unless some special case occurs, when it is $2 m$ ) whose component at $v$ is $\chi^{v}$. The effectiveness problem for this theorem is to bound the norm $N(\chi)$ of the conductor of $\chi$ in terms of $K, m, S$ and $N\left(\chi^{v}\right)$.

The Kummer case (when $K$ contains $\mu_{m}$ ) is easy since it is almost an application of the Chinese Remainder Theorem. In this note, we solve this problem completely in general case, and give three versions of bound, one is with GRH, and the other two are unconditional bounds.

These effective results have some interesting applications in concrete situations. To give a simple example, if we fix $p$ and $l$, one gets a good least upper bound for $N$ such that $p$ is not an $l$-th power $\bmod N$. One also gets the least upper bound for $N$ such that $l^{r} \mid \phi(N)$ and $p$ is not an $l$-th power $\bmod N$.

Some part of this note is adopted (with some revision) from my unpublished thesis (2001) ([26]).
\end{abstract}

\section{Contents}

1. Introduction

2. Preliminaries

2.1. Grunwald-Wang

2.2. Estimations

3. The Variant and the Proof 10

3.1. The Variant 11

3.2. Main Arguments and Proofs 12

3.3. Estimations again 16

4. The $S$-effective Version of the Strong Multiplicity One - GL(1) Case 18

4.1. Proof (B) 18

4.2. $S$-version of Multiplicity One for $G L(n) \quad 22$

References $\quad 22$

The author was supported in part by national 973 project 2013CB834202, also National Natural Science Foundation of China (NNSFC 11321101), and also by the One Hundred Talent's Program from Chinese Academy of Science. 


\section{INTRODUCTION}

Let $K$ be a number field, $K_{v}$ the local completion of $K$ at $v$, with multiplicative group $K_{v}^{\times}$. $\mathbb{A}_{K}$ the ring of adeles of $K, \mathbb{I}_{K}=\mathbb{A}_{K}^{\times}$the group of ideles, and $C_{K}=$ $\mathbb{I}_{K} / K^{\times}$the group of idele classes.

In 1933, W. Grunwald ([5]) stated a striking theorem asserting that, given a finite set $S$ of places of $K$, and a family of characters $\chi^{v}(v \in S)$ of $K_{v}^{\times}$of orders $m_{v}$ (which are also called local characters), there exists a continuous character $\chi$ of finite order of $C_{K}$ (which is also called a global character) whose local component $\chi_{v}$ at each $v \in S$, which are obtained by composing $\chi$ with the natural local embedding $K_{v}^{\times} \rightarrow C_{K}$, is exactly $\chi^{v}$. Furthermore, the order of $\chi$ can be made as the least common multiple $m$ of the $m_{v}$.

However, Grunwald's original statement and proof had a flaw, which occurred when he discussed the special case. The gap was filled by Sh. Wang ([29, [30]), who also gave a precise criterion for the special case and showed that the order of $\chi$ can be taken to be $m$ under an additional condition (see Section 2 and also Chapter 10, [1]). So this theorem is appropriately called the Grunwald-Wang theorem.

Given the local datum $\left\{\chi^{v} \mid v \in S\right\}$, there are infinitely many global characters $\chi$ with local components $\chi^{v}$. They can be highly ramified in general. Indeed, some additional ramification has to be allowed as seen for example even when all the $\chi^{v}$ are unramified and $K$ has class number 1 . (If we remove the order requirement of $\chi$, then there exists a $\chi$ with given local behavior, and more over, $\chi$ is unramified outside all finite places outside $S$.) However, the natural question which arises is whether we can control the ramification of $\chi$ in terms of $K, S, m$ and the norms $N\left(\chi^{v}\right)$ of the conductors of $\chi_{v}$ for $v$ in $S$.

Unfortunately, the original proof by Grunwald and Wang was not effective, and did not answer this question. This effectiveness problem was first encountered in 1995 in the work of J. Hoffstein and D. Ramakrishnan on Siegel zeros ([ 6$]$ ), where they needed it in a particular case when $K \supset \mu_{m}$ and $m$ a prime (the Kummer case). As roughly (not precisely) each character of $K_{v}\left(\right.$ or $\mathbb{I}_{K}=\mathbb{A}_{K}^{\times}$) of exponent $m$ corresponds to a Kummer extension $K_{v}\left(\sqrt[m]{y_{v}}\right) / K_{v}$ or $K(\sqrt[m]{y}) / K$ by the Kummer theory, the problem can be converted directly into the situation when the Chinese Remainder Theorem applies (see [26]). Although such method is extendable to also the general Kummer case ( $K$ contains $\mu_{m}$ ) and also the case when $K$ is general and $m$ is an odd prime, it fails in the general case. So the question in general is not easy.

In the thesis [26, we solved this problem completely. we are going to explain our main results. Without loss of generality, may assume that $m=l^{r}$ a prime power since it is easy for us to reduce to this case since any local/global character of finite order can be written uniquely as a product of characters of prime power degree.

Now we introduce some notations. For each place $v$ of $K$, let $\mathfrak{p}_{v}$ be the symbol of the formal prime of $K$ corresponding to $v$, which can be used also as the prime idea corresponding to $v$ of $\mathcal{O}_{K}$ or $\mathcal{O}_{K_{v}}$, the ring of integers of $K$ or $K_{v}$ respectively (depending on the context around). Let $\chi_{v}$ ( $\chi$ resp. ) be a continuous character of $K_{v}^{\times}\left(C_{K}\right.$ resp. $)$. Define the arithmetic conductor or norm as the following: 


$$
N\left(\chi_{v}\right)= \begin{cases}1 & \left(\chi_{v} \text { is unramified or } v \text { is real or complex }\right) \\ q_{v}^{n} & \left(n \text { is the smallest integer such that }\left(1+\mathfrak{p}_{v}^{n}\right)^{\times} \subset \operatorname{Ker}\left(\chi_{v}\right)\right)\end{cases}
$$

where $q_{v}$ is 1 if $v$ is archimedean and the size of residue field of $K_{v}$ when $v$ is finite.

$$
N(\chi)=\prod_{v} N\left(\chi_{v}\right)
$$

Moreover, $N_{S}=\prod_{v \in S} q_{v}$, and $n_{K}=[K: \mathbb{Q}]$. Also denote $S_{\infty}$ be the set of infinite places of $K$. Moreover, for each finite set $T$ of places of $K$ containing all infinite ones, denote $\mathrm{C} \ell_{K, T}$ the $T$-class group of $K$ and $c \ell_{K, T}$ its size.

By convention, for two quantities $f$ and $g$ depending on various parameters, $f<<g$ or $f=O(g)$ means $f<C g$ for some absolute positive constant, and $f<<_{v, v^{\prime}, \ldots g}$ or $f=O_{v, v^{\prime}, \ldots}(g)$ means $f<C g$ for some constant $C$ only depending on $v, v^{\prime}, \ldots$ which are parameters shown up in the subscript.

Theorem-Definition A. Let $K$ be a number field, $m=l^{r}$ a prime power, $S$ a finite set of places of $K, \chi^{v}$ a local character of $K_{v}^{\times}$of exponent $m$ is given for each $v \in S$. Assume $K\left(\zeta_{l^{r}}\right) / K$ is cyclic, or the special case occurs with some specified condition holds (See Section 2). There is a global character $\chi$ of $C_{K}$ of exponent $m$ with its local parts $\chi_{v}=\chi^{v}$ for all $v \in S$.

Moreover, denote BPI the smallest number of $N(\chi)$ for such $\chi$.

Of course, BPI $<\infty$ as when $K, S, m=l^{r}$ is given, the number of the choice of $\left\{\chi^{v}, v \in S\right\}$ is finite.

In 26] (unpublished), we show that

$$
\mathbf{B P I} \leq A \prod_{v \in S} N\left(\chi^{v}\right)^{B}
$$

with $A=\left(A_{0} N_{S}\right)^{C_{1}|S|}$ where $A_{0}, C_{1}$ are constants only depending on $K$ and $m$, not on $S$, and moreover for the special case when $K \supset \mu_{m}$ OR $m$ is a prime We also proved for a better bound $A=A_{0} N_{S}^{C_{2}}$, where $A_{0}, B, C_{1}$ and $C_{2}$ are independent of $S$. The later bound improves the result of [6]. The bound BPI in this paper is improved compared with [26].

Theorem A.1. All notations are as in Theorem $A$, We have

$$
\log \mathbf{B P I}<<l^{r}\left(\left|S \cup S_{\infty}\right|+D\right) \log \left(d_{K} N_{S} l^{n_{K} r}\right)
$$

where $D=\gamma_{l}\left(\mathrm{C} \ell_{K, S \cup S_{\infty}}\right)$, the minimal size of generating set of the Sylow l-subgroup of $\mathrm{C} \ell_{K, S \cup S_{\infty}}$, which is also the $\mathbb{F}_{l}$-rank of $\mathrm{C} \ell_{K, S \cup S_{\infty}}[l], l$-torsion part of the class group $\mathrm{C} \ell_{K, S \cup S_{\infty}}$.

Theorem A.2. All notations as in Theorem $A$ and Theorem A.1. We have

$$
\mathbf{B P I}<<_{\epsilon, K, l, r} N_{S}^{E_{1}(1 / 2+\epsilon)} \prod_{v \in S} N\left(\chi^{v}\right)
$$

where

$$
E_{1}=\left[K\left(\zeta_{l^{r}}\right): K\right]\left(\left|S \cup S_{\infty}\right|+D-\delta^{\prime}\right)+\delta
$$


and $D=\gamma_{l}\left(\mathrm{C} \ell_{K, S \cup S_{\infty}}\right)$ and

$$
\begin{aligned}
& \delta= \begin{cases}0 & \left(K\left(\zeta_{l^{r}}\right)=K\right) \\
1 & \left(K\left(\zeta_{l^{r}}\right) \neq K\right)\end{cases} \\
& \delta^{\prime}= \begin{cases}1 & \left(\zeta_{l} \notin K .\right) \\
0 & \text { (otherwise. })\end{cases}
\end{aligned}
$$

For example, when $K=\mathbb{Q}, m=l^{r}$ and $S=\{p\}$, we have $\mathrm{C} \ell_{K, S \cup S_{\infty}}=1$ and hence $D=0$. Moreover, $\delta^{\prime}=0$ if $l=2$ and 1 if $l$ is odd; $\delta=0$ if $l^{r}=2,1$ if $l^{r}=4$ or odd prime power and 2 if $l=2$ and $r \geq 3$.

$$
E_{1}= \begin{cases}l^{r-1}(l-1)+1 & (l \text { is odd. }) \\ 2 & \left(l^{r}=2 .\right) \\ 2^{r}+1 & (l=2, r \geq 2 .)\end{cases}
$$

(Note that if $2 \notin S$ and $r \geq 3$, then we can replace $\delta=1$ by 0 .)

The following theorem is a result with GRH (General Riemann Hypothesis) (which is an analogue of Riemann Hypothesis to the case of general $L$-functions).

Theorem A.3. All notations as in Theorem A and Theorem A.1. Also assume GRH holds. Then there is an effectively computable $C>0$ only depending on $l, r$ such that

$$
\mathbf{B P I} \leq\left(C \log \left(d_{K} N_{S} l^{n_{K}}\right)\right)^{2\left(\left|S \cup S_{\infty}\right|+D-\delta^{\prime}+\delta\right)} \prod_{v \in S} N\left(\chi^{v}\right)
$$

where $D=\gamma_{l}\left(\mathrm{C} \ell_{K, S \cup S_{\infty}}\right), \delta$ and $\delta^{\prime}$ are defined in Theorem A.1 and Theorem A.2.

Remark: $\delta=1$ can be replaced by 0 in the following two cases: (1) $\zeta_{l^{r}} \notin K$, $K\left(\zeta_{l^{r}}\right) / K$ is cyclic, and $S_{l} \not \subset S,(2) l=2$ and $K\left(\zeta_{2^{r}}\right) / K$ is not cyclic and $S_{0} \not \subset S$.

Remark: In fact, the quantity $\left|S \cup S_{\infty}\right|+D-\delta^{\prime}$ is exactly the $\mathbb{F}_{l}$-rank of the Selmer group $\operatorname{Sel}_{l}^{S}(K)$.

These effective results have some interesting applications in concrete situations. To give a simple example, if we fix $p$ and $l$, one gets a good least upper bound for $N$ such that $p$ is not an $l$-th power $\bmod N$. One also gets the least upper bound for $N$ such that $l^{r} \mid \phi(N)$ and $p$ is not an $l$-th power $\bmod N$.

Let's explain the idea of these theorems right now. There are two main ingredients. First, we are trying to reduce this effective problem to a variant. Roughly speaking, Let $P=\prod_{v \in S} K_{v}^{\times} \subset \mathbb{I}_{K}$ and $P_{0}=\operatorname{Ker} \prod_{v} \chi^{v} \subset P$. To find the global $\chi$ with given local behavior, it suffices to find a suitable standard open subgroup $V$ of $\mathbb{I}_{K}$ such that

$$
K^{\times} \mathbb{I}_{K}^{m} V \cap P \subset P_{0} \quad(*)
$$

This is exactly how the Grunwald-Wang was proved in [1. To find the least bound for $N(\chi)$, it suffices to choose $V=\prod_{v} V_{v}$ carefully to ensure $\left(^{*}\right)$ (Of course for $v \in S$ we choose $V_{v}$ lying in the kernel of $\left.\chi^{v}\right)$. Let $\gamma i^{m} u \in P$ where $i \in \mathbb{I}_{K}, \gamma \in K^{\times}$ and $u=\left(u_{v}\right) \in V$. Then $m \mid v(\gamma)$ for $v \notin S$ finite. Then $\gamma=\gamma^{\prime} c^{m}$ for some $\gamma^{\prime}$ lies in certain finite generate abelian subgroup $A$ of $K^{\times}$. Choose a finite set $T$ of finite places of $K$, disjoint from $S$, such that $A \cap \bigcap_{v \in T}\left(1+\mathfrak{p}_{v}\right) \subset A^{m}$. Now let $V_{v}=1+\mathfrak{p}_{v}$ when $v \in T$, and $V_{v}=\mathcal{O}_{v}$ outside $T \cup S$, and hence such $V$ meets our need. We 
are able to do that since $A$ is finite generated. (In fact, $A[l]$, the $l$-torsion part of such $A$ is isomorphic to the Selmer group

$$
\operatorname{Sel}_{l}^{S}(K)=\left\{[x] \in K^{S} / K^{S^{l}}, x \in K_{v}^{\times l} \forall v \in S\right\}
$$

)

Now, pick any $\alpha \in A-A^{m}$, we can choose $v$ outside $S$ such that $v$ is inert at $K\left(\zeta_{m}, \sqrt[m]{\alpha}\right) / K\left(\zeta_{m}\right)$ so that $\alpha \notin K_{v}^{\times^{m}}$. A series $v$ can ensure that $A \cap \bigcap_{v}\left(1+\mathfrak{p}_{v}\right) \subset$ $A \cap K\left(\zeta_{m}\right)^{\times m}$. Then add at most two more $v$ to make the intersection to $A^{m}$. Over $v$ s $K\left(\zeta_{m}, \sqrt[m]{A}\right) / K$ does not collapse, i.e., $\forall x \in K\left(\zeta_{m}, \sqrt[m]{A}\right)$, if $x \in K_{v}$ for all $v \in T$ then $x \in K$ (See section 2).

Now the second ingredient comes. To find $v$ and bound the norm as above, we are always in the following situation: Let $E / F$ be a Galois extension, and we want to fine the least prime ideal $\mathfrak{p}$ of $F$ outside a given finite set $S$ not split in $E$. Some $S$ version of Chebotarev Density Theorems (more precisely, $S$-version of Multiplicity One Theorems in $G L(1)$, see Section 3 and 4) will answer this question. Our three main theorem rely on exactly three $S$-versions of multiplicity one theorems (also see Theorem 3.11, Theorem 4.1).

\section{Theorem 1.1. ( $S$-version of the Multiplicity One for $G L(1)$, Theorem 4.1)}

Let $K$ be a number field and $\chi$ a nontrivial global character of $C_{K}$ of finite order. Then there is a place $v$ of $K$ such that

(1) $\mathfrak{p}_{v} \notin S$.

(2) $\chi_{v} \neq 1$ and is not ramified.

(3 A) $\log N\left(\mathfrak{p}_{v}\right)<<\log A(\chi, S)$

(3 B) $N\left(\mathfrak{p}_{v}\right)<<_{\epsilon, K} N(\chi)^{1 / 2+\epsilon} N_{S}^{\epsilon}$ for every $\epsilon>0$.

(3 C) With GRH, $N\left(\mathfrak{p}_{v}\right)<<(\log A(\chi, S))^{2}$.

where $A(\chi, S)=d_{K} N(\chi) N_{S}$.

In fact, (A) and (C) are $S$-effective versions of [9] and [10, and the proof runs through some variant of the proof of the above two papers (For details, see [27]). (B), uses Landau's method, and runs arguments like [13] with some modifications. We include the proof in Section 4 .

This paper is organized as the following: In Section 2, after the notation, we introduce the Grunwald-Wang Theorem, and the special case of Wang. Experts can skip most parts of this section. In Section 3 we will formulate and prove the variant and hence our theorems. As mentioned before, three $S$-version of multiplicity one for $G L(1)$ will be formulated there and will be proved in Section 4 .

Most parts of Section 3 and some parts of Section 4 are adopted from Chapter 5 of my thesis [26] with certain revision. Theorem A.2 is new since it adopts results from other people in later publications ([2, [28, [13). Here we express again our great appreciation for my advisor D. Ramakrishnan for his suggestion of this topic, and for the continued help during my graduate study and continuing years. 


\section{Preliminaries}

In this part, we'll recall the Grunwald-Wang Theorem, and also make some preliminaries. First we recall some notations.

Again, $K$ denotes a number field or a function field in this section, $S$ a finite set of places of $K$. For each place $v, K_{v}$ denotes the completion of $K$ at $v$ which is a complete discrete valuation field. When $v$ is non-archimedean, denote $\mathcal{O}_{K_{v}}$ the valuation ring of $K_{v}$ and $\mathcal{U}_{K_{v}}=\mathcal{O}_{K, v}^{\times}$the unit group of $K_{v}$.

Denote $\mathbb{A}_{K}$ the ring of Adeles of $K, \mathbb{I}_{K}=\mathbb{A}_{K}^{\times}$the group of ideles of $K$. Denote $C_{K}=\mathbb{I}_{K} / \times K^{\times}$the idele class group of $K$. When $S$ contains $S_{\infty}$, the set of all archimedean places of $K$ which is empty when $K$ is a function field, denote $\mathbb{A}_{K, S}$ and $\mathbb{I}_{K, S}=\mathbb{A}_{K, S}^{\times}$the ring of $S$-adeles and the group of $S$-ideles respectively. Recall that $\mathbb{A}_{K, S}=\prod_{v \in S} K_{v} \times \prod_{v \notin S} \mathcal{O}_{K_{v}}$ and $\mathbb{A}_{K}=\bigcup_{S \supset S_{\infty}} \mathbb{A}_{K, S} . K$ and $K^{\times}$embed into $\mathbb{A}_{K}$ and $\mathbb{I}_{K}$ diagonally respectively. Denote $K^{S}=K^{\times} \cap \mathbb{I}_{K, S}$ the set of $S$-units (which are those $a \in K$ such that $a \in \mathcal{U}_{K_{v}}$ ) for $v \notin S$.

\subsection{Grunwald-Wang.}

First recall the following results which is crucial for the statement and the proof of the Grunwald-Wang Theorem. Denote $\zeta_{n}$ a primitive root of unity of the order $n$, and we choose $\zeta_{n}$ for all $n$ to ensure that $\zeta_{n m}^{m}=\zeta_{n}$. Denote $\eta_{n}=\zeta_{n}+\zeta_{n}^{-1}$.

\section{Proposition 2.1. ([1, Theorem 10.1)}

Let $P(m, S)$ be the set of all $x \in K^{\times}$such that $x \in\left(K_{v}^{\times}\right)^{m}$ for all $v \notin S$.

Then $P(m, S)=K^{\times^{m}}$, unless exactly if the following conditions hold. (If they hold, we say that the special case of Wang occurs.)

(a) $K$ is a number field.

(b) $-1, \pm\left(2+\eta_{2^{s}}\right)$ are non-squares in $K^{\times}$where $s$ is the integer such that $\eta_{2^{s}} \in$ $K^{\times}, \eta_{2^{s+1}} \notin K^{\times}$.

(c) $m=2^{t} m^{\prime}, 2 \nmid m^{\prime}, t \supsetneqq s$,

(d) $S_{0} \subseteq S$, where $S_{0}$ is the set of places $v \mid 2$ such that $-1, \pm\left(2+\eta_{2^{s}}\right)$ are nonsquares in $K_{v}$.

In the special case, $P(m, S)=K^{\times m} \cup a_{0} K^{\times^{m}}$, where $a_{0}=\left(1+\zeta_{2^{s}}\right)^{m}=\eta_{2^{s+1}}^{m}=$ $\left(i \eta_{2^{s+1}}\right)^{m}=\left[ \pm\left(2+\eta_{2^{s}}\right)\right]^{m / 2}$.

Remark: When the special case of Wang occurs, $F=K\left(\sqrt{-1}, \sqrt{2+\eta_{2^{s}}}\right)$ is a $F_{4}=\mathbb{Z} / 2 / b Z \times \mathbb{Z} / 2 \mathbb{Z}$ extension of $K$, and for all $v$ such that $K_{v}\left(\sqrt{-1}, \sqrt{2+\eta_{2^{s}}}\right) / K_{v}$ is also an $F_{4}$-extension, $v \in S_{0}$. In particular, $t \geq 3$.

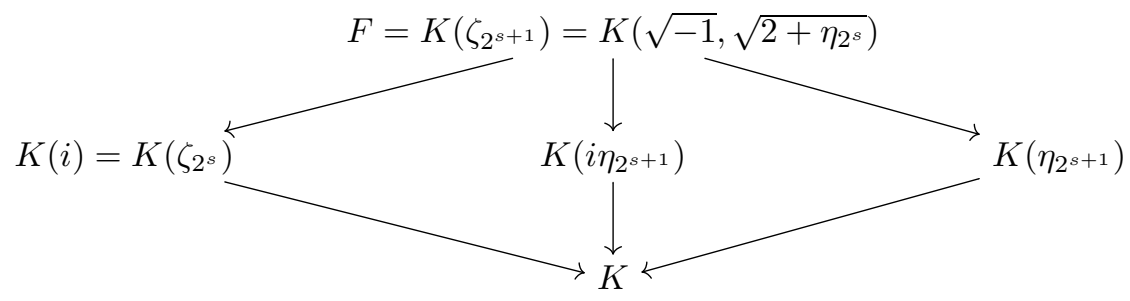


Remark: The simplest special case of Wang is the following: $K=\mathbb{Q}, m=8$, $S=\{2\}$ and $a_{0}=16$. One simple example of the special case of Wang when $S=\infty$ is the following: $K=\mathbb{Q}(\sqrt{7}), m=8$ and $a_{0}=16$.

Now we state the first version of the Grunwald-Wang Theorem. Recall that a character is said to be of exponent $m$ if its order divides $m$.

Theorem 2.2. ([1], Theorem 10.5-1)

Let $S$ be a finite set of places of $K$, and $\chi^{v}$, a local character of $K_{v}^{\times}$of exponent $m$ for each $v \in S$.

Then there is a global character $\chi$ of $C_{K}$ of exponent $m$ with its local parts $\chi_{v}=\left.\chi\right|_{K_{v}^{\times}}=\chi^{v}$ for all $v \in S$ unless the special case of Wang (Proposition 2.1) occurs.

When the special case of Wang occurs, such $\chi$ can still be chosen of exponent $m$ when the following condition holds

$$
\prod_{v \in S} \chi_{v}\left(a_{0}\right)=1
$$

where $a_{0}$ is defined as in Proposition 2.1. Otherwise, such $\chi$ can be chosen of exponent $2 \mathrm{~m}$.

By the class field theory, the local and global version, we have the following variants which is the corollary.

Theorem 2.3. ([1], Theorem 10.5-2)

Let $S$ be a finite set of places of $K$, and $L^{v} / K_{v}$ a cyclic extension of degree $m_{v}$ for each $v \in S$.

Then there is a cyclic extension $L / K$ of degree $m=\operatorname{LCM}\left(m_{v}\right)$, the least multiple of $m_{v}$ for $v \in S$, such that $L_{w} \cong L^{v}$ for the place $w$ of $L$ above $v$ for $v \in S$ unless the special case of Wang (Proposition 2.11) occurs.

When the special case of Wang occurs, such $L$ can still be chosen of degree $2 m$ over $K$.

In next section we will formulate a variant. It is known that this variant implies Theorem 2.2 (1]). We will prove this variant in an effective way.

\subsection{Estimations.}

In this part, we list some results of estimations.

Proposition 2.4. (Discriminant-Conductor Formula) ([10, [18])

Let $L / K$ be an abelian extension of local (resp. number fields) of degree $n$, and $\chi_{0}=1, \chi_{1}, \ldots, \chi_{n-1}$ be all $n$ local character (resp. idele class characters) of $K^{\times} / N_{L / K}\left(L^{\times}\right) C_{K} / N_{L / K} C_{L}$. Then

$$
d_{L}=d_{K}^{n} \prod_{i=1}^{n-1} N\left(\chi_{i}\right)
$$


Remark: By the class field theory, $\operatorname{Gal}(L / K) \cong K^{\times} / N_{L / K} L^{\times}(\operatorname{resp} \cdot \operatorname{Gal}(L / K) \cong$ $C_{K} / N_{L / K} C_{L}$ ) when $L / K$ is an abelian extension of local fields (resp. number fields) of degree $n$, and hence there are exactly $n=[L: K]$ characters.

Lemma 2.5. Let $L / K$ be an abelian extension of local fields (resp. number fields) of prime degree $l$, and $\chi$ a local character (resp. idele class character) associated to $L / K$. Then

$$
d_{L}=d_{K}^{l} N\left(\chi_{i}\right)^{l-1}
$$

Proof: This is a corollary to the previous lemma.

Recall that $N_{S}$ denotes the set of norms of (formal) primes $\mathfrak{p}_{v}$ for all $v \in S$. Recall that

$$
N\left(\mathfrak{p}_{v}\right)= \begin{cases}1 & (v \text { is real and complex. }) \\ q_{v}=\#\left(\mathcal{O}_{K_{v}} / \mathfrak{p}_{v}\right) & (v \text { is finite. })\end{cases}
$$

Now we define the conductor cycle $\mathfrak{f}\left(\chi_{v}\right)$ of a local character $\chi_{v}$ of $K_{v}^{\times}$(of finite order) as following:

$$
\mathfrak{f}\left(\chi_{v}\right)= \begin{cases}1 & \left(\chi_{v}=1, v \text { is real or complex. }\right) \\ \mathfrak{p}_{v} & \left(\chi_{v}=\text { Sgn, } v \text { is real. }\right) \\ 1 & \left(\chi_{v} \text { is unramified, } v \text { is finite. }\right) \\ \mathfrak{p}_{v}^{n} & \left(\chi_{v} \text { is ramified, and } n \text { is the smallest }\right) \\ & \left(\text { integer such that } \chi_{v}\left(1+\mathfrak{p}_{v}^{n}\right)=1\right)\end{cases}
$$

and $N\left(\chi_{v}\right)=N\left(\mathfrak{f}\left(\chi_{v}\right)\right)$.

A formal cycle of $K$ is a formal product $\prod_{v} \mathfrak{p}_{v}^{n_{v}}$ where $n_{v}=0,1$ when $v$ is real, $n_{v}=0$ when $v$ is complex, $n_{v} \geq 0 \in \mathbb{Z}$ for other $v$, and $n_{v}=0$ for almost all $v$. Define the norm of $\mathfrak{f}=\prod_{v} \mathfrak{p}_{v}^{n_{v}}=\prod_{v}\left(N\left(\mathfrak{p}_{v}\right)\right)^{n_{v}}$.

For a idele class character $\chi$ of finite order, denote its conductor cycle $\mathfrak{f}(\chi)=$ $\prod_{v} \mathfrak{f}\left(\chi_{v}\right)$ where $\chi_{v}$ is the restriction of $\chi$ to $K_{v}^{\times}$. Denote $\mathfrak{f}_{\mathrm{f}}(\chi)=\prod_{v \text { finite }} \mathfrak{f}\left(\chi_{v}\right)$. Define the arithmetic conductor or the norm of $\chi$ as $N(\chi):=N(\mathfrak{f}(\chi))$, the norm of its conductor cycle, and the analytic conductor or level $A(\chi)$ of $\chi$ as $A(\chi):=$ $d_{K} N(\chi)$.

Lemma 2.6. Let $K_{v}$ be a local field with valuation $v$ and assume that $p$ is the characteristic of its residue field. Then if $v(x)>v(m)+\frac{e_{v / p}}{p-1}$ where $e_{v / p}=v(p)$ the ramification index of $K_{v} / \mathbb{Q}_{p}$, then $1+x \in \mathcal{O}_{K_{v}}^{\times}{ }^{m}$. In addition, if $p \nmid m$ and $v(x)>0$, then $1+x \in \mathcal{O}_{K_{v}}^{\times}$.

Proof: Want to prove that under this condition the binomial series $(1+x)^{1 / m}=$ $\sum_{k=0}^{\infty}\left(\begin{array}{c}1 / m \\ k\end{array}\right) x^{k}$ converges. Let $a_{n}=\left(\begin{array}{c}1 / m \\ k\end{array}\right) x^{k}$ where

$$
\left(\begin{array}{l}
N \\
k
\end{array}\right)=\frac{N(N-1) \cdots(N-k+1)}{k !}
$$




$$
\begin{aligned}
v\left(a_{k}\right) & =v\left(\frac{1}{m}\left(\frac{1}{m}-1\right) \cdots \frac{1}{m}+k+1\right)-v(k !)+k v(x) \\
& \geq k(v(x)-v(m))-v(k !) \\
& \geq k\left(v(x)-v(m)-e_{v / p}(p-1)\right) \\
& \rightarrow 0 \quad(k \rightarrow \infty)
\end{aligned}
$$

Here the second inequality holds since the multiplicity of the factor $p$ in $k$ ! is $[k / p]+\left[k / p^{2}\right]+\cdots$ which is not greater than $k / p-1$.

For the case when $p \nmid m$, the assertion follows since we can prove that $\left(\begin{array}{c}1 / m \\ k\end{array}\right)$ a $p$-adic integer.

Lemma 2.7. (1) If $\chi$ is a global character of $C_{K}$ of order $m$, then

$$
\mathfrak{f}_{\mathrm{f}}(\chi) \text { divides }(m) \prod_{p \mid m}(p) \prod_{v \in S_{\mathrm{Ram}}} \mathfrak{p}_{v}
$$

where $S_{\mathrm{Ram}}$ is the set of finite places $v$ where $\chi_{v}=\chi_{K_{v}^{\times}}$is ramified. Moreover,

$$
N(\chi) \leq\left(m \prod_{p \mid m} p\right)^{n_{K}} N_{S_{\text {Ram }}}
$$

where $n_{K}=[K: \mathbb{Q}]$.

(2) In particular, if $m=l^{r}$ where $r>0$, then

$$
N(\chi) \leq l^{n_{K}(r+1)} N_{S_{\text {Ram }}} \leq l^{n_{K}(r+2)} N_{S_{\text {Ram }}-S_{l}}
$$

where $S_{l}$ is the set of places $v$ of $K$ such that $v \mid l$.

Proof:

(1) Assume $\mathfrak{f}_{\mathrm{f}}(\chi)=\prod_{v \in S_{\mathrm{Ram}}} \mathfrak{p}_{v}^{n_{v}}$. From Lemma 2.6 $n_{v}=1$ if $v \nmid m$. If $p \mid m$ and $v \mid p$, then $n_{v} \leq 1+v(m)+e_{v / p} /(p-1) \leq 1+v(m)+v_{p}=1+v(p m)$. Thus

$$
\begin{array}{r}
\mathfrak{f}_{\mathrm{f}}(\chi)=\prod_{v \in S_{\mathrm{Ram}}}(\chi) \prod_{p \mid m} \prod_{v \mid p} \mathfrak{p}_{v}^{v(p m)} \\
\text { divides } \prod_{v \in S_{\mathrm{Ram}}}(\chi)(m) \prod_{p \mid m}(p)
\end{array}
$$

Take the norm, we get the last assertion in (1). (2) is routine to check when we take $m=l^{r}$.

Remark: In Lemma 2.7(2), when $l$ is odd, then we can have

$$
N(\chi) \leq l^{n_{K} r} N_{S_{\text {Ram }}}
$$

instead. This follows from Lemma 2.6 (2) also by checking carefully. 
Lemma 2.8. (1) Let $K^{\prime}=K\left(\zeta_{l^{r}}\right)$. Then

$$
d_{K^{\prime}} \leq\left(l^{n_{K}(r+2)}\right)^{\left[K^{\prime}: K\right]-1} d_{K}^{\left[K^{\prime}: K\right]}
$$

(2) Let $\tilde{\chi}$ be a global character of $C_{K^{\prime}}$ of order $l$ associated to the Kummer extension $K^{\prime}(\sqrt[l]{a}) / K^{\prime}$ via the global class field theory where $a \in K^{\times}$. Let $T_{a}$ be the set of finite places $v$ of $K$ such that $v \nmid l$ and $l \nmid v(a)$. Then

$$
N(\tilde{\chi}) \leq l^{n_{K^{\prime}}(r+2)} N_{T_{a}}^{\left[K^{\prime}: K\right]}=\left(l^{n_{K}(r+2)} N_{T_{a}}\right)^{\left[K^{\prime}: K\right]}
$$

and

$$
A(\tilde{\chi}) \leq\left(l^{n_{K} 2(r+2)} d_{K} N_{T_{a}}\right)^{\left[K^{\prime}: K\right]}
$$

(3) Let $\chi^{\prime}$ be a global character of $C_{K}$ of order $l$ associated to the subextension of the cyclic extension $K\left(\zeta_{l^{r}}\right) / K$. Then

$$
N\left(\chi^{\prime}\right) \leq l^{3 n_{K}}
$$

and

$$
A\left(\chi^{\prime}\right) \leq l^{3 n_{K}} d_{K}
$$

Proof: Let $n^{\prime}=\left[K^{\prime}: K\right]$ where $K^{\prime}=K\left(\zeta_{l^{r}}\right)$.

(1): Let $\chi_{0}=1, \chi_{1}, \ldots, \chi_{n^{\prime}-1}$ be the $n^{\prime}$ characters of $C_{K} / N_{L / K} C_{L}$. From Proposition 2.4 the Discriminant-Conductor formula, we have $d_{K^{\prime}} \leq D_{K}^{n^{\prime}} \prod_{i=1}^{n-1} N\left(\chi_{i}\right)$. Now all assertions follow from Lemma 2.6 (2), since all $\chi_{i}$ are ramified at finite places $v \nmid l$, as by the class field theory, the Galois extension $K_{i}^{\prime} / K$ associated to $\chi_{i}$ are all subextensions of the cyclotomic extensions $K^{\prime}=K\left(\zeta_{l^{r}}\right) / K$.

(2): Note that the following fact: For each $a \in K^{\times}$, the Kummer extension $K^{\prime}(\sqrt[l]{a}) / K^{\prime}$ is ramified at $v \nmid l$ if and only if $l \nmid v(a)$, hence $\tilde{T}^{\prime}$, the set of places of $K^{\prime}$ not dividing $l$ ramify in this Kummer extension, is contained in the set of places $w$ of $K^{\prime}$ above $T_{a}$. Hence $\tilde{T}^{\prime} \leq N_{T_{a}}^{\left[K^{\prime}: K\right]}$.

Now it is routine to check (2) from Lemma 2.7(1) and (2).

(3): Clear from Lemma $2.7(2)$.

\section{The Variant and the Proof}

In this section, we will formulate a variant of the Grunwald-Wang Theorem. Also, we'll prove this variant and then the main theorems of this paper. 


\subsection{The Variant.}

Now $K$ again denotes a number field, and $S$ a finite set of places of $K$. Let $P=$ $\prod_{v \in S} K_{v}^{\times} \hookrightarrow \mathbb{I}_{K}$ endowed with the induced topology from $\mathbb{I}_{K}, P_{0}$ a subgroup of $P$ containing $P^{m}$. When the special case of Wang occurs, denote $\mathrm{a}_{0}=\left(a_{0}, \ldots a_{0}\right) \in P$ where $a_{0} \in K^{\times}$is defined as in Proposition 2.1.

\section{Theorem 3.1. ([1, Theorem 10.4)}

Let $S, P, P_{0}, \mathbf{a}_{0}$ as above. Then their exists an open subgroup $V$ of $\mathbb{I}_{K}$ such that $K^{\times} \mathbb{I}_{K}^{n} V \cap P \subset P_{0}$ where $n$ is an integer such that $K^{\times} \mathbb{I}_{K}^{n} \cap P \subset P_{0}$. Moreover, $n$ can be taken as $m$ unless the special case of Wang occurs. When the special case of Wang holds, then $n$ can be taken as $m$ when $\mathbf{a}_{0} \in P_{0}$. Otherwise, $n$ be taken as $2 m$.

Remark: As proven in [1], Theorem 3.1 implies the Grunwald-Wang since when given $\left\{\chi^{v}, v \in S\right\}$, we can set $P_{0}=\operatorname{Ker} \prod_{v} \chi^{v}$, and $N=P_{0} \mathbb{I}_{K}^{m} K^{\times} V$, then $P / P_{0} \cong$ $\mathbb{I}_{K} / N$ through the natural embedding $i_{P}: P \hookrightarrow \mathbb{I}_{K}$. Thus $\chi$ can be chosen as

$$
\chi: \mathbb{I}_{K} / K^{\times} \rightarrow \mathbb{I}_{K} / N \stackrel{i_{P}^{-1}}{\longrightarrow} P / P_{0} \stackrel{\prod_{v \in S} \chi^{v}}{\longrightarrow} \mathbb{C}^{\times}
$$

Before we formulate the effective version of the variant, we recall some definitions. Let $\mathfrak{c}=\prod_{v} \mathfrak{p}_{v}^{n_{v}}$ be a formal cycle of $K$. Define the standard open subgroup $V_{\mathfrak{c}}$ as $\prod_{v} \mathcal{U}_{v}^{\left(n_{v}\right)}$ where

$$
\begin{aligned}
\mathcal{U}_{v}^{(0)}=K_{v}^{\times}(v \text { is real or complex }) \\
\mathcal{U}_{v}^{(1)}=\mathbb{R}^{+, \times}(v \text { is real }) \\
\mathcal{U}_{v}^{(0)}=\mathcal{U}_{K_{v}}(v \text { is finite }) \\
\mathcal{U}_{v}^{(n)}=1+\mathfrak{p}_{v}^{n} \subset \mathcal{U}_{K_{v}}(v \text { is finite, } n \geq 1)
\end{aligned}
$$

Theorem-Definition B. Let $K$ be a number field, $m=l^{r}$ a prime power, $S$ a finite set of places of $K, P=\prod_{v \in S} K_{v}^{\times} \hookrightarrow \mathbb{I}_{K}, P_{0}$ a subgroup of $P$ containing $P^{m}$. Assume that $K^{\times} \mathbb{I}_{K}^{m} \cap P \subset P_{0}$.

Then there is a standard open subgroup $V_{\mathfrak{c}}$ of $\mathbb{I}_{K}$ such that $K^{\times} \mathbb{I}_{K}^{m} V_{\mathfrak{c}} \cap P \subset P_{0}$.

Denote $\mathbf{B P V}$ be the smallest number of $N(\mathfrak{c})$ for such $V_{\mathfrak{c}}$.

The main theorems of this paper (Theorem A.1 A.2, A.3) follow from the following theorems.

Theorem 3.2. All notations are same as in Theorem-Definition $A$ and $B$, Then $\mathrm{BPI} \leq \mathrm{BPV}$.

Theorem 3.3. All notations are as in Theorem $B$. We have

$$
\log \mathbf{B P V}<<l^{r}\left(\left|S \cup S_{\infty}\right|+D\right) \log \left(d_{K} N_{S} l^{n_{K} r}\right)
$$

where $D=\gamma_{l}\left(\mathrm{C} \ell_{K, S \cup S_{\infty}}\right)$, the minimal size of generating set of the Sylow l-subgroup of $\mathrm{C} \ell_{K, S \cup S_{\infty}}$. 
Theorem 3.4. All notations as in Theorem B, Theorem 3.3. We have

$$
\mathbf{B P V}<<_{\epsilon, K, l, r} N_{S}^{E_{1}(1 / 2+\epsilon)} N\left(\mathfrak{c}_{0}\right)
$$

where

$$
E_{1}=\left[K\left(\zeta_{l^{r}}\right): K\right]\left(\left|S \cup S_{\infty}\right|+D-\delta^{\prime}\right)+\delta
$$

where $D=\gamma_{l}\left(\mathrm{C} \ell_{K, S \cup S_{\infty}}\right)$ and

$$
\begin{aligned}
& \delta= \begin{cases}0 & \left(K\left(\zeta_{l^{r}}\right)=K\right) \\
1 & \left(K\left(\zeta_{l^{r}}\right) \neq K\right)\end{cases} \\
& \delta^{\prime}= \begin{cases}1 & \left(\zeta_{l} \notin K .\right) \\
0 & \text { (otherwise. })\end{cases}
\end{aligned}
$$

and $\mathfrak{c}_{0}$ is the smallest cycle such that $V_{\mathfrak{c}} \cap P \subset P_{0}$.

Remark: When $P / P_{0}$ is cyclic, and $P_{0}=\prod_{v \in S} \chi^{v}$, then $\mathfrak{c}_{0}=\prod_{v \in S} \mathfrak{f}\left(\chi^{v}\right)$.

Remark: $\delta=1$ can be replaced by 0 in the following two cases: (1) $\zeta_{l^{r}} \notin K$, $K\left(\zeta_{l^{r}}\right) / K$ is cyclic, and $S_{l} \not \subset S,(2) l=2$ and $K\left(\zeta_{2^{r}}\right) / K$ is not cyclic and $S_{0} \not \subset S$. This also applies to Theorem 3.5 .

Theorem 3.5. All notations as in Theorem B, Theorem 3.3 and Theorem 3.4. Also assume GRH holds. Then there is an effectively computable $C>0$ only depending on $l, r$ such that

$$
\mathbf{B P V} \leq\left(C \log \left(l^{n_{K}} d_{K} N_{S}\right)\right)^{2\left(\left|S \cup S_{\infty}\right|+D-\delta^{\prime}+\delta\right)} N\left(\mathfrak{c}_{0}\right)
$$

where $D=\gamma_{l}\left(\mathrm{C} \ell_{K, S \cup S_{\infty}}\right), \delta, \delta^{\prime}$ and $\mathfrak{c}_{0}$ are defined in Theorem $B$ and Theorem 3.4.

Theorem 3.2 follows from the remark after Theorem-Definition B. The rest of this paper will be devoted to the proof of these theorems.

\subsection{Main Arguments and Proofs.}

In this part, we will prove Theorem B, 3.3, 3.4, 3.5 and finally the main theorem. Again recall the notation and our situation. $K$ denotes a number field, $m=l^{r}$ a prime power, $S$ a finite set of finite places. Let $K^{\prime}=K\left(\zeta_{l^{r}}\right)$. Recall that $P=\prod_{v \in S} K_{v}^{\times}$, and $P \supset P_{0} \supset P^{m}$. To make the discussion easier, we may assume that $K^{\times} \mathbb{I}_{K}^{m} \cap P \subset P_{0}$ (which means that the special case of Wang occurs and the special condition to guarantee the order $m$ also holds, see Theorem 3.1). The goal is to find the least bound $\mathbf{B P V}$ of $N(\mathfrak{c})$ for $V_{\mathfrak{c}}$ such that $K^{\times} \mathbb{I}_{K}^{m} V \cap P \subset P_{0}$ (cf. Theorem 3.1).

Definition: Let $E / F$ be an abelian extension of number fields. Let $T$ be a finite set of places of $F$. Denote $B(E / F, T, l)$ be the least bound $C$ such that, for each subextension $E^{\prime}$ of $E / F$ cyclic of degree $l$, there is a prime $\mathfrak{p}_{v}$ of $F$ satisfying: (a) $v \notin T$. (b) $\mathfrak{p}_{v}$ is unramified and does not split in $E^{\prime}$. (c) $N\left(\mathfrak{p}_{v}\right) \leq C$.

Remark: By the Chebotarev Density Theorem, $B(E / F, T, l)<\infty$.

Definition: Define $P^{*}(n, S)$ as the set of $x \in K^{\times}$such that $m \mid v(x)$ for each $v \notin S$.

Moreover, recall that for a finite abelian group $H, \gamma_{l}(H)$ denotes the minimal generators of the Sylow-l group 
Proposition 3.6. Let $E / F$ be an abelian l-extension of number fields, $T$ a finite set of places of $K$. Let $e=\gamma_{l}(\operatorname{Gal}(E / F))$. Then There are e places $w_{1}, w_{2}, \ldots, w_{e}$ of $F$ such that

(1) $w_{i} \notin T$ for all $1 \leq i \leq e$.

(2) For any $a \in E-F$, their is a wi which does not split in $F(a) / F$.

(3) $N\left(\mathfrak{p}_{w_{i}}\right) \leq B(E / F, T, l)$.

Proof: We proceed in steps.

(Step 1): Assume first that $E / F$ is an elementary $l$-extension, i.e., $\operatorname{Gal}(E / F) \cong$ $\mathbb{F}_{l}^{e}$. Change (2) to the following (2)':

Induct in $e . e=1$ is OK. Assume that the proposition holds for $<e$, and now want to prove the $e$ case.

Pick a cyclic subextension $E_{1} / F$, which is cyclic of degree $l$, and pick $w_{1} \notin T$ of degree 1 , where $E_{1} / F$ is inert at $w_{1}$, and moreover $N\left(\mathfrak{p}_{w_{1}}\right) \leq B\left(E_{1} / F, T, l\right) \leq$ $B(E / F, T, l)$.

Let $E_{2}$ be the fixed field of $D_{w_{1}}\left(E_{1} / F\right)$, the decomposition group of $E / F$ at $w_{1}$. Then $\left[E_{2}: F\right]=l^{e-1}$, and $w_{1}$ splits in $E_{2} / F$ completely. By induction, there are $w_{2}, w_{3}, \ldots, w_{e}$ satisfying (1), (2), (3) with $E$ replaced by $E_{2}$.

Now, (1) and (3) hold for original $E$ and $w_{1}, w_{2}, \ldots, w_{e}$. For (2), for each $a \in$ $E-F$, if $a \in E_{2}$, then (2) for $E^{\prime}$ and $w_{2}, \ldots, w_{e}$ implies (2). If $a \notin E_{2}$, then $D_{w_{1}}(E / F)$ does not fix $a$, and hence $w_{1}$ does not split in $F(a) / F$ then.

(Step-2): Assume that $E / F$ is an $l$-extension. Let $E^{\prime}$ be the composition of elementary $l$-subextensions of $E / F$. Thus $\operatorname{Gal}\left(E^{\prime} / F\right) \cong \mathbb{F}_{l}^{e}$. Let $w_{1}, w_{2}, \ldots, w_{e}$ be chosen to satisfying (1), (2), (3) for $E^{\prime}$. Then (1), (3) for $E$ holds. For (2) for $E$, let $a \in E-F$, want to find $w_{i}$ such that $F(a) / F$ is not split at $w_{i}$. In fact, the maximal elementary $l$-subextension of $F(a) / F$ is not split at $w_{i}$ for some $i$, and hence $F(a) / F$ is not split at this $w_{i}$.

Proposition 3.7. Let $K^{\prime}=K\left(\zeta_{l^{r}}\right)$. Let $e=\left|S \cup S_{\infty}\right|+D-\delta^{\prime}$. Assume that $K^{\prime} / K$ is cyclic.

Then there exist places $v_{1}, v_{2}, \ldots, v_{e+\delta}$ of $K$ satisfying the following:

(1) $v_{1}, v_{2}, \ldots, v_{e+\delta} \notin S$.

(2) For any $y \in P^{*}\left(l^{r}, S\right)$ such that $y \in K_{v_{i}}^{\times l^{r}} \quad(1 \leq i \leq e+\delta)$, we have $y \in K^{\times l^{r}}$.

(3) $N\left(\mathfrak{p}_{v_{i}}\right) \leq B\left(K^{\prime}\left(\sqrt[l^{r}]{P^{*}\left(l^{r}, s\right)}\right) / K, \tilde{S}, l\right)$ for $1 \leq i \leq e$ and $N\left(\mathfrak{p}_{v_{e+1}}\right) \leq B\left(K^{\prime} / K, S, l\right)$ if $\delta=1$.

Here $\tilde{S}$ the set of places of $K^{\prime}$ above the places in $S, D=\gamma_{l}\left(\mathrm{C} \ell_{K, S \cup S_{\infty}}\right)$ and

$$
\begin{aligned}
& \delta= \begin{cases}0 & \left(K\left(\zeta_{l^{r}}\right)=K\right) \\
1 & \left(K\left(\zeta_{l^{r}}\right) \neq K\right)\end{cases} \\
& \delta^{\prime}= \begin{cases}1 & \left(\zeta_{l} \notin K .\right) \\
0 & \text { (otherwise. })\end{cases}
\end{aligned}
$$


Remark: This is the effective version of Proposition 2.1.

Proof: Recall $m=l^{r}$.

(Step-1): Let $\tilde{K}=K^{\prime}\left(\sqrt[l^{r}]{P^{*}\left(l^{r}, S\right)}\right)$. Then $\gamma_{l}\left(\operatorname{Gal}\left(\tilde{K} / K^{\prime}\right)\right) \leq e$.

Let $T$ be a set of places containing $S \cap S_{\infty}$ such that $\mathrm{C}_{K, T}$ is not divisible by l. We can choose $T$ such that $|T|=\left|S \cup S_{\infty}\right|+D$ where $D=\gamma_{l}\left(\mathrm{C} \ell_{K, S \cup S_{\infty}}\right)$. In particular, if $l \nmid \mathrm{C} \ell_{K, S \cup S_{\infty}}$ then $D=0$. In fact, let $Q$ be the Sylow $l$-group of $\mathrm{C} \ell_{K, S \cup S_{\infty}}$ and $c_{1}, \ldots, c_{D}$ be a set of generators of $Q$. By the Chebotarev density, there exist places $w_{1}, \ldots, w_{D}$ of $K$ such that the ideal class $\mathfrak{p}_{w_{j}}$ in $\mathrm{C} \ell_{K, S \cup S_{\infty}}$ is $c_{j}$ for each $1 \leq j \leq D$.

Let $T=S \cup S_{\infty} \cup\left\{w_{1}, \ldots w_{D}\right\}$. Then $P^{*}(m, S) \subset K^{\times m} K^{T}$. In fact, for each $y \in P^{*}(m, S)$, let $\mathfrak{a}_{y}=\prod_{v \notin S \cap S_{\infty}} \mathfrak{p}_{v}^{v(y) / l^{r}}$. Then the ideal $\mathfrak{a}_{y}^{l^{r}}=(y) J$ for some $J \in J_{K}^{S}$ where $J_{K}^{S}$ is the ideal group generated $\mathfrak{p}_{v}$ for $v \in S$. Hence the class of $\mathfrak{a}_{y}$ in $\mathrm{C} \ell_{K, S \cup S_{\infty}}$ has power $l^{r}$. Hence by the choice of $T$, the class of $\mathfrak{a}_{y}$ in $\mathrm{C} \ell_{K, T}$ is trivial. In particular, there is $z \in K^{\times}$such that $\mathfrak{a}_{y}=(z) \mathfrak{a}^{\prime}$ where $\mathfrak{a}^{\prime}$ is some ideal in $J_{K}^{T}$. Thus $y z^{-l^{r}} \in K^{T}$. Hence $\tilde{K} \subset \tilde{K}^{\prime}=K^{\prime}\left(\sqrt[l^{r}]{K^{T}}\right)$. Thus

$$
\gamma_{l}\left(\tilde{K} / K^{\prime}\right) \leq \gamma_{l}\left(\tilde{K}^{\prime} / K^{\prime}\right) \leq \operatorname{rank}_{\mathbb{F}_{l}} K^{T} /\left(K^{T}\right)^{l}
$$

Now (Step-1) is done $\operatorname{since} \operatorname{rank}\left(K^{T}\right)=\left|S \cup S_{\infty}\right|-1+D$ (and hence $\tilde{K} / K^{\prime}$ is a finite abelian extension, and

$$
\operatorname{rank}_{\mathbb{F}_{l}}\left(K^{T} /\left(K^{T}\right)^{l}\right)=e=\left|S \cup S_{\infty}\right|+D-\delta^{\prime}
$$

(Step-2): Assume that $K^{\prime}=K$, i.e., $\zeta_{l^{r}} \in K$. Then by Proposition 3.6 there exist places $w_{1}, \ldots, w_{e}$ of $K$ of outside $S$, such that, for each $b \in \tilde{K}-K, K(b) / K$ is not split at $w_{i}$ for some $i$, and moreover $N\left(\mathfrak{p}_{w_{i}}\right) \leq B(\tilde{K} / K, S, l)$ (by (Step-1)).

Now consider any $y \in P^{*}(m, S)$ such that $y \in K^{\times^{m}}$. Set $b \in \sqrt[l^{r}]{y}$, and by the discussion above, $K(b) / K$ is not split for some $w_{i}$, and hence $b \notin K$. Hence $y \notin K^{\times^{m}}$. Hence $v_{1}=w_{1}, \ldots, v_{e}=w_{e}$ are what we want.

(Step-3): $K^{\prime} \neq K$ and $\delta=1$. By Proposition 3.6, there exist places $w_{1}, \ldots, w_{e}$ of $K^{\prime}$ of outside $\tilde{S}$, such that, for each $b \in \tilde{K}-K^{\prime}, K^{\prime}(b) / K^{\prime}$ is not split at $w_{i}$ for some $i$, and moreover $N\left(\mathfrak{p}_{w_{i}}\right) \leq B\left(\tilde{K} / K^{\prime}, \tilde{S}, l\right)$ (by (Step-1)). Moreover, there exists a place $v_{1}^{\prime}$ of $K$ outside $S$, such that $K^{\prime} / K$ is inert at $v_{1}^{\prime}$, and moreover $N\left(\mathfrak{p}_{v_{i}^{\prime}}\right) \leq B\left(K^{\prime} / K, S, l\right)$ (Proposition 3.6 noting that fact $\gamma_{l}\left(K^{\prime} / K\right)=1$ ).

Now let $v_{1}, \ldots, v_{e}$ be the places of $K$ under $w_{1}, \ldots, w_{e}$ and $v_{j+1}=v_{j}^{\prime}(j \leq 1)$. Then (1) and (3) are clear.

For (2), Let $y \in P^{*}(m, S)$ and assume that for all $i=1, \ldots e+\delta, y \in K_{v_{i}}^{\times^{m}} \subset$ $K_{w_{i}}^{\prime \times m}$. Hence by the choice of $w_{1}, \ldots, w_{e}$, we have $y \in K^{\prime \times^{m}}$.

let $K_{1}$ be the maximal $l$-subextension of $K^{\prime} / K$. First claim $y \in K_{1}^{\times^{m}}$. In fact, $y^{\left[K^{\prime}: K_{1}\right]}=N_{K^{\prime} / K_{1}}(y) \in K_{1}^{\times m}$, and hence $y \in K_{1}^{\times m}$ since $l \nmid\left[K^{\prime}: K_{1}\right]$ and $\left(\left[K^{\prime}: K_{1}\right], m\right)=1$. As $\left[K_{1}: K\right]$ is a cyclic $l$-extension, then it has only one elementary $l$-subextension $K_{2}$. By the choice of $v_{e+1}=v_{1}^{\prime}, K_{2} / K$ is not split at $v_{1}^{\prime}$ and $K_{1} / K$ is inert at $v_{1}^{\prime}$. Since $y \in K_{v_{1}^{\prime}}^{\times m}$, there is a $z \in K^{\prime}$ such that $z^{m}=y$, and $z \in K_{v_{1}^{\prime}}$, and hence $K(z)=K$ as $K(z) / K$ is inert at $v_{1}^{\prime}$. Hence $y \in K^{\times m}$. 
Lemma 3.8. Let $m=2^{r}$ and assume that $K^{\prime}=K\left(\zeta_{m}\right) / K$ is not cyclic. Let $K^{+}=K\left(\eta_{2^{m}}\right)$. Let $s$ be the integer such that $\eta_{2^{s}} \in K$ but $\eta_{2^{s+1}} \notin K$.

(1) Let $y \in K(i)$ or $K\left(i \eta_{2^{s+1}}\right)$ such that $y^{m} \in K^{\times}$, then there is an $j \in \mathbb{Z}$ such that $y\left(1+\zeta_{2^{s}}\right)^{j} \in K$.

(2) Denote $a_{0}=\left(1+\zeta_{2^{s}}\right)^{m}$. Assume that $v$ is a place such that $\eta_{2^{s+1}} \notin K_{v}$, and $Y \in K_{v}^{\times m} \cap K^{\times} \cap K^{\prime \times m}$. Then $Y \in K^{\times m} \cup a_{0} K^{\times m}$.

(3) Let $v \mid 2$ be such that -1 and $\pm\left(2+\eta_{2^{s}}\right)$ is not square in $K_{v}^{\times}$. Assume $Y \in$ $K_{v}^{\times^{m}} \cap K^{\times} \cap K^{\prime \times m}$ then $Y \in K^{\times^{m}}$.

Proof: In this case $s<r$. Moreover, $\left[K^{\prime}: K^{+}\right]=2$. Let $\sigma$ be the nontrivial $K^{+}$automorphism of $K^{\prime}$. Then $\sigma\left(\zeta_{2^{r}}\right)=\zeta_{2^{r}}^{-1}$. Moreover $K\left(\zeta_{2^{s+1}}\right) / K$ is a 4-extension with three intermediate subextension $K(i), K\left(\eta_{2^{s+1}}\right)$ and $K\left(i \eta_{2^{s+1}}\right)$.

For more analysis on this case, see Section 3 .

(1) Put $y_{r}=y\left(1+\zeta_{2^{s}}\right)^{r}$. Then $y_{r} / \sigma\left(y_{r}\right)=(y / \sigma(y)) \zeta_{2^{s}}^{r}$. Want to prove that $y^{2^{s}}=\sigma\left(y^{2^{s}}\right)$ so that, can pick $r$ such that $\sigma\left(y_{r}\right)=y_{r}$ and hence $y_{r} \in K$ as $K=K(i)^{\sigma}=K\left(i \eta_{2^{s+1}}\right)^{\sigma}$.

Note that $T=y / \sigma(y)$ is a root of unity in $K(i)$ or $K\left(i \eta_{2^{s+1}}\right)$. Hence $T=\zeta_{s}^{j}$ for some $j$ in the first case or $T= \pm 1$ in the later case. As $s \geq 2$, in all case we have $T^{s}=1$. Hence such $j$ is what we want.

(2) By the choice of $v, K^{\times} / K$ is inert at $v$. Then either $K(i)$ or $K\left(i \eta_{2^{s+1}}\right)$ is split at $v$. Let $K_{2}$ be such field, and $w$ a place above $v$. By comparing the residue degree, $K^{\prime} / K_{2}$ is inert at $w$ as they have the same residue degree as $K^{+} / K$. Thus $Y \in K_{v}^{\times m}=K_{2, w}^{\times}{ }^{m}$. Hence there is some $z$ lies in $K^{\prime} \cap K_{v}=K_{2, w}$ such that $z^{m}=y$. As $K^{\prime} / K_{2}$ is inert at $w, z \in K_{2}$. Thus (2) follows from (1) since $\left(1+\zeta_{2^{s}}\right)^{m}=a_{0}$.

(3) There is a $z \in K^{\prime} \cap K_{v}$ such that $z^{m}=y$. By the choice of $v$ and the analysis on $K^{\prime} / K$ at the beginning, $K^{\prime} / K$ does not collapse at $v$. Hence $z \in K$ and $Y=z^{m} \in K^{\times^{m}}$.

Proposition 3.9. Let $K^{\prime}=K\left(\zeta_{2^{r}}\right)$ and $e=\left|S \cup S_{\infty}\right|+D$. Assume that $K^{\prime} / K$ is not cyclic. Denote $s, K^{+}$and $a_{0}$ are as in Lemma 3.8. Let $S_{0}$ be the set of places $v \mid 2$ where -1 and $\pm\left(2+\eta_{2^{s}}\right)$ is not a square in $K_{v}^{\times}$.

Then there exist places $v_{1}, v_{2}, \ldots, v_{e+1}$ of $K$ satisfying the following:

(1) $v_{1}, v_{2}, \ldots, v_{e+1} \notin S$. If $S_{0} \not \subset S$ then $v_{e+1} \in S_{0}-S$.

(2) For any $y \in P^{*}\left(2^{r}, S\right)$ such that $y \in K_{v_{i}}^{\times^{2}} \quad(1 \leq i \leq e+1)$, we have $y \in K^{\times 2^{r}}$ if $S_{0} \not \subset S$ and $y \in K^{\times 2^{r}} \cup a_{0} K^{\times 2^{r}}$ if $S_{0} \subset S$.

(3) $N\left(\mathfrak{p}_{v_{i}}\right) \leq B\left(K^{\prime}\left(\sqrt[r^{r}]{P^{*}\left(2^{r}, s\right)}\right) / K, \tilde{S}, 2\right)$ for $1 \leq i \leq \delta$ and $N\left(\mathfrak{p}_{v_{e+1}}\right) \leq B\left(K^{\prime} / K, S, 2\right)$ if $S_{0} \subset S$.

Here $\tilde{S}$ the set of places of $K^{\prime}$ above the places in $S, D=\gamma_{2}\left(\mathrm{C} \ell_{K, S \cup S_{\infty}}\right)$.

Proof: Recall $m=2^{r}$. Note that (Step-1) of the proof of Proposition 3.7 still applies since we don't use the assumption that $K\left(\zeta_{l^{r}}\right) / K$ is cyclic. Moreover, (Step$3)$ goes through smoothly except for the verifications of (2). 
(Step-3'): Let $v_{1}, \ldots, v_{e}$ be the places of $K$ under $w_{1}, \ldots, w_{e}$ as in (Step-3) the proof of Proposition 3.6 and $v_{j+\delta}=v_{j}^{\prime}(j \leq \delta)$ if $S_{0} \subset S$ and any place in $S_{0}-S$ if $S_{0} \not \subset S$. Then again (1) and (3) are clear.

For (2), Let $y \in P^{*}(m, S)$ and assume that for all $i=1, \ldots e+1, y \in K_{v_{i}}^{\times^{m}} \subset$ $K_{w_{i}}^{\prime \times m}$. Hence by the choice of $w_{1}, \ldots, w_{e}$, we have $y \in K_{w_{i}}^{\prime}{ }^{m}$ and hence $y \in$ $K^{\prime x^{m}}$.

Now (2) follows from Lemma 3.8

Theorem 3.10. All notations are as in Theorem-Definition B, Theorem 3.1, Theorem 3.2. Proposition 3.7 and Proposition 3.9, Then

$$
\mathbf{B P V} \leq l^{n_{K}(r+2)} N\left(\mathfrak{c}_{0}\right) B\left(K^{\prime}\left(\sqrt[l]{P^{*}\left(l^{r}, S\right)}\right) / K^{\prime}, \tilde{S}, l\right)^{\left|S \cup S_{\infty}\right|+D-\delta^{\prime}} B\left(K^{\prime} / K, S, l\right)^{\delta}
$$

where $\mathfrak{c}_{0}$ is the smallest cycle such that $V_{\mathfrak{c}} \cap P \subset P_{0}$.

Proof: Again denote $e=\left|S \cup S_{\infty}\right|+D-\delta^{\prime}$. If we are in the special case of Wang, let $\mathbf{a}_{0}=\left(a_{0}\right) \in P$ be the idele with component $a_{0}$ at all $v \in S$ and 1 elsewhere.

Let $v_{1}, \ldots, v_{e+\delta}$ be the set of places chosen in Proposition 3.7 (resp. Proposition 3.9) when $K^{\prime}=K\left(\zeta_{l^{r}}\right)$ is cyclic (resp. $K^{\prime} / K$ is not cyclic).

Now consider three cycles as following: $\mathfrak{c}_{0}$ the conductor cycle of $P_{0}$ as in Theorem 3.5. Theorem 3.6 and Theorem 3.7. $\mathfrak{c}_{1}=(l)^{r+1} \prod_{v \mid l} \mathfrak{p}_{v}$ and $\mathfrak{c}_{2}=\prod_{i=1}^{e+\delta}\left(\mathfrak{p}_{v_{i}}\right)$ Let $\mathfrak{c}=\mathfrak{c}_{0} \mathfrak{c}_{1} \mathfrak{c}_{2}$, and we have $N(\mathfrak{c})$ is bounded by the right hand side of the formula in this theorem (Proposition 3.7, Proposition 3.9).

Want to prove $K^{\times} \mathbb{I}_{K}^{m} V_{\mathfrak{c}} \cap P \subset P_{0}$. Let $\gamma \in K^{\times}, B=\left(B_{v}\right) \in \mathbb{I}_{K}$ and $u=\left(u_{v}\right) \in V_{\mathfrak{c}}$ such that $Y=\gamma B^{m} u \in P$. Want $Y \in P_{0}$.

In fact, $\gamma B_{v}^{m} u_{v}=1$ for $v \notin S, \gamma \in P^{*}(m, S)$. Moreover, for each $v=v_{i}$, if $v \nmid l$ (resp. if $\left.v_{i} \mid l\right)$, then $U_{v}=1+\mathfrak{p}_{v} \subset K_{v}^{\times m}$ (resp. $U_{v}=1+\mathfrak{p}_{v}^{(r+1) v(l)+1} \subset K_{v} \times$ (Lemma 2.5, 2.7)). Hence $\gamma \in K_{v_{i}}^{\times m}$ for $i=1, \ldots e+\delta$.

Thus by Proposition 3.7 and Proposition 3.9. we have $\gamma \in K^{\times m}$, or $\gamma \in K^{\times^{m}} \cup$ $a_{0} K^{\times m}$ when we are in the special case of Wang and $S_{0} \subset S$.

When $\gamma \in K^{\times^{m}}$, then for $v \in S, Y_{v}=\gamma B_{v}^{m} u_{v} \in u_{v} K_{v}^{\times^{m}}$, and hence $Y \in$ $\left(P \cap V_{\mathfrak{c}_{0}}\right) P^{m} \subset P_{0}$.

When we are in the special case of Wang and $\gamma \in K^{\times m} \cup a_{0} K^{\times m}$, we have for each $v, Y_{v} \gamma B_{v}^{m} u_{v} \in u_{v}\left(K_{v}^{\times^{m}} \cup a_{0} K_{v}^{\times m}\right)$, and hence $Y \in\left(P \cap V_{\mathfrak{c}_{0}}\right)\left(P^{m} \cup \mathbf{a}_{0} P^{m}\right) \subset P_{0}$ as $\mathbf{a}_{0} \in P_{0}$. Done.

3.3. Estimations again. In this part, we'll plug in all estimations we made in Section 1 to finish the proof.

We have the following three effective versions of the Chebotarev density and multiplicity one, which will be proved in next section.

For each global character $\chi$ of $C_{K}$, define $A(\chi, S):=A(\chi) \prod_{v \in S, \chi_{v} \text { is unramified }} \leq$ $A(\chi) N_{S}$. 
Theorem 3.11. There is a prime $\mathfrak{p}_{v}$ satisfying the following:

(1) $\mathfrak{p}_{v} \notin S$.

(2) $\chi_{v} \neq 1$ is unramified.

(3) $N\left(\mathfrak{p}_{v}\right)$ has the following versions of the least bound:

(A) $\log \left(N\left(\mathfrak{p}_{v}\right)\right)<<\log A(\chi, S)$.

(B) $N\left(\mathfrak{p}_{v}\right)<<_{\epsilon, K} N(\chi)^{1 / 2+\epsilon} N_{S}^{\epsilon}$.

(C) Assuming GRH, $N\left(\mathfrak{p}_{v}\right)<<(\log A(\chi, S))^{2}$.

For the proof, see Section 4

Corollary 3.12. Let $L / K$ be a cyclic extension of degree $m$. There is a prime $\mathfrak{p}_{v}$ satisfying the following:

(1) $\mathfrak{p}_{v} \notin S$.

(2) $\mathfrak{p}_{v}$ is ramified and does not split in $L / K$.

(3) Let $\chi$ be the global character of $C_{K}$ corresponding to $L / K$ via the class field theory. $N\left(\mathfrak{p}_{v}\right)$ has the exactly three versions of the least bound as the same in (3) in Theorem 3.11.

Lemma 3.13. (1) Let $K^{\prime}=K\left(\zeta_{l^{r}}\right)$ and $\tilde{K}=K^{\prime}\left(\sqrt[l^{r}]{P^{*}\left(l^{r}, S\right)}\right)$. Let $\tilde{S}$ be as in Proposition 3.7 and Proposition [3.9, and $\tilde{\chi}^{\prime}$ a global character associated to a cyclic subextension of $\tilde{K} / K^{\prime}$. of order $l$. Then $A\left(\tilde{\chi}^{\prime}, \tilde{S}\right) \leq\left(l^{2 n_{K}(r+2)} d_{K} N_{S}\right)^{\left[K^{\prime}: K\right]}$.

(2) Let $\chi^{\prime}$ be a global character associated to a subextension of $K^{\prime} / K$ of order $l$. Then $A\left(\chi^{\prime}, S\right) \leq\left(l^{3 n_{K}}\right) d_{K} N_{S}$.

Proof: (1) Any subextension of $K^{\prime} / K$ is a Kummer extension. So applying Lemma 2.8 (2) by noting that $T_{a} \subset S$. (2) follows from Lemma 2.8 (3).

Proof of Theorem 3.3. Theorem 3.4 and Theorem 3.5 using Theorem 3.10 and Theorem 3.11]

Proof: We proceed in three situation:

(1) Theorem 3.3

$$
\begin{aligned}
\log \mathbf{B P V} \leq \log N\left(\mathfrak{c}_{0}\right)+e \log B\left(\tilde{K} / K^{\prime}, \tilde{S}, l\right)+\delta \log B\left(K^{\prime} / K, S, l\right) \\
\quad \leq \log \left(N_{S}\right)+\left(n_{K}(r+2)\right) \log (l)+e\left[K^{\prime}: K\right] \log l^{2 n_{K}(r+2)} d_{K} N_{S} \\
\quad+\delta \log l^{3 n_{K}} d_{K} N_{S} \\
\quad<<\left(e\left[K^{\prime}: K\right]+\delta\right) \log \left(l^{r n_{K}} d_{K} N_{S}\right) \\
\quad<<l^{r}\left(\left|S \cup S_{\infty}\right|+D\right) \log \left(l^{r n_{K}} d_{K} N_{S}\right)
\end{aligned}
$$

(2) Theorem 3.4 and Theorem 3.5 Can be proved similarly by using Theorem 3.10 and Theorem 3.11 
Proof of Theorem A.1, Theorem A.2. Theorem A.3.

Proof: Use Theorem 3.2, Theorem 3.3, Theorem 3.4, Theorem 3.5

4. The $S$-effective Version of the Strong Multiplicity One - GL(1)

CASE

In this section, we'll prove Theorem 3.11 and Corollary 3.12 .

First we summarize the rough idea of Theorem 3.11 (A) and (C). The main idea of them is the same as in [10] and 9. Theorem 3.11 (A) (without GRH) and (C) (with GRH) follows from a variant of Theorem 1.2 and Corollary 1.3 (plus its proof) in 9. The only difference is that we replace the quantities $d_{L}, A(\chi)$ by $d_{L}^{\prime}=d_{L} N_{S}^{[L: K]}$ and $A(\chi, S)=A(\chi) N_{S}$. More details can be also found in [27].

In this section we prove (B). which uses Landau's idea (see $[8$, the proof follows almost from [13]).

Now we restate Theorem 3.11 (A) and (B) as the following theorems.

Theorem 4.1. ( $S$-version of the Multiplicity One for $G L(1)$ )

Let $K$ be a number field and $\chi$ a nontrivial global character of $C_{K}$ of finite order. Then there is a place $v$ of $K$ such that

(1) $\mathfrak{p}_{v} \notin S$.

(2) $\chi_{v} \neq 1$ and is not ramified.

(3 A) $\log N\left(\mathfrak{p}_{v}\right)<<\log A(\chi, S)$

(3 B) $N\left(\mathfrak{p}_{v}\right)<<_{\epsilon, K} N(\chi)^{1 / 2+\epsilon} N_{S}^{\epsilon}$ for every $\epsilon>0$.

(3 C) With GRH, $N\left(\mathfrak{p}_{v}\right)<<(\log A(\chi, S))^{2}$.

where $A(\chi, S)=d_{K} N(\chi) N_{S}$.

\subsection{Proof (B). :}

In this part, we prove Theorem 4.1 (which is Theorem $3.11(\mathrm{~B})$ ). We'll quote a lot of standard results of number theory (for references of textbook style see 11, [12, [17, [19], 33] etc, for related topics, see [2], 6], 9], [10, 28], [13, [20, [26], 23 etc). To make things easier, may assume that for all $v \in S$, if $v$ is a finite place, and $\chi_{v}$ is unramified. In this case $A(\chi, S)=A(\chi) N_{S}$.

Proof of Theorem 4.1.

Set

$$
S(X, \chi, S)=\sum_{n=1}^{\infty} a_{\chi, S}(n) \omega\left(\frac{n}{X}\right)
$$

where $a_{\chi, S}(n)$ is the $n$-th coefficient of the Hecke $L$-function $L^{S}(s, \chi)=$ $\prod_{v \notin S}\left(1-\chi_{v}\left(\pi_{v}\right) N\left(\mathfrak{p}_{v}\right)^{-s}\right)^{-1}$ 
$=\sum_{n=1}^{\infty} a_{\chi, S}(n) n^{-s}$, and the weight function $\omega(x)$ defined as a smooth function which may be specified as [28]:

$$
\omega(X)= \begin{cases}0 & (x \leq 0 \text { or } x \geq 3) \\ e^{-1 / x} & (0<x \leq 1) \\ e^{-1 /(3-x)} & (2 \leq x<3) \\ \leq 1 & (\text { all } x)\end{cases}
$$

Consider the Mellin transform

$$
W(s)=\int_{0}^{\infty} \omega(x) x^{s-1} d x
$$

which is a analytic function of $s$. Fix $\sigma<0$ and let $s=\sigma+i t$ then

$$
W(s)<<_{A, \sigma} \frac{1}{(1+|t|)^{A}}
$$

for all $A>0$ by repeated partial integration. By Mellin inversion,

$$
\omega(x)=\frac{1}{2 \pi i} \int_{(2)} W(s) x^{-s} d s
$$

where the integration is made along the vertical line Res $=2$.

Then we have

$$
\begin{aligned}
S(X, \chi, S) & =\frac{1}{2 \pi i} \sum_{n=1}^{\infty} a_{\chi, S}(n) \int_{(2)} W(s)\left(\frac{n}{X}\right)^{-s} d s \\
& =\frac{1}{2 \pi i} \int_{(2)} X^{s} W(s) L^{S}(s, \chi)
\end{aligned}
$$

where the interchange of the summation and the integral is guaranteed by the absolute convergence along the real line $\sigma=2$. By the standard arguments, plus the fact that all incomplete $L$-functions of nontrivial characters are entire of order 1 , we may shift the integral line to get

$$
S(X, \chi, S)=\frac{1}{2 \pi i} \int_{-H} x^{s} W(s) L^{S}(s, \chi) d s
$$

where $H>0$ is to be specified later.

Let $L_{S}(s, \chi)=\prod_{v \in S} L\left(s, \chi_{v}\right)$, and $L_{\infty}(s, \chi)=L\left(s, \chi_{\infty}\right)$ the gamma factor of $\chi$. Then we have the following functional equation

$$
L^{S}(s, \chi)=W(\chi) A(\chi)^{(1 / 2-s)} G_{0}(s) G_{1}(s) L^{S}\left(1-s, \chi^{-1}\right)
$$

where $W(\chi)$ is the root number of $\chi$ which has absolute value 1 ,

$$
G_{0}(s)=\frac{L_{\infty}\left(1-s, \chi^{-1}\right)}{L_{\infty}(s, \chi)}
$$

and

$$
G_{1}(s)=\frac{L_{S}\left(1-s, \chi^{-1}\right)}{L_{S}(s, \chi)}
$$

We need to estimate $G_{0}(s)$ and $G_{1}(s)$ along the vertical line $\sigma=-H$, avoiding to the pole of them. 
Lemma 4.2. (1) If $H>0$ is not an integer, then

$$
G_{0}(-H+i T)<<_{H, n_{K}}(1+|t|)^{n_{K}(1 / 2+H)}
$$

(2) $G_{1}(-H+i T)<<_{H, n_{K}, \epsilon^{\prime}} N_{S}^{H+\epsilon^{\prime}}$.

We quote the following results on gamma function for which the proof can be found in a lot of analysis textbooks.

\section{Lemma 4.3. (Stirling formula)}

$$
|\Gamma(\sigma+i t)|=\sqrt{2 \pi} e^{-\frac{\pi}{2} t}|t|^{\sigma-1 / 2}\left(1+O_{\sigma, \delta, A}\left(\frac{1}{1+|t|}\right)\right)
$$

for all $|t|>A>0$ and $s=\sigma+i$ away from any poles by at least distance $\delta$.

Proof of Lemma 4.2.

(1) It is well known that there are $a(\chi)$ and $b(\chi)$ be the integer such that $L_{\infty}(s, \chi)=C \pi^{-s n_{K} / 2}(\Gamma(s / 2))^{a(\chi)}(\Gamma((s+1) / 2))^{b(\chi)}$ and $a(\chi)+b(\chi)=n_{K}$. Note that $a(\chi)=a\left(\chi^{-1}\right)$. Now assume $H>0,|T|>1$, then

$$
\begin{aligned}
G_{0}(-H+i T) & =\frac{L_{\infty}\left(1+H-i T, c h i^{-1}\right)}{L_{s}(-H+i T, \chi)} \\
& =\pi^{n_{K}(-1 / 2-H+i T)} \frac{\Gamma((1+H-i T) / 2)}{\Gamma((-H+i T) / 2)} \frac{\Gamma((H-i T) / 2)}{\Gamma((1-H+i T) / 2)} \\
& <<_{H, n_{K}}\left(\frac{|T|^{H / 2}}{|T|^{-(1+H) / 2}}\right)^{a(\chi)}\left(\frac{|T|^{(H+1) / 2}}{|T|^{-H / 2}}\right)^{b(\chi)} \\
& <<_{H, n_{K}}|T|^{n_{K}(1 / 2+H)} \\
& <(1+|T|)^{n_{K}(1 / 2+H)}
\end{aligned}
$$

where we apply the Stirling (Lemma 4.3) in the third row since the poles of the gamma factors are non-positive integers. For $0 \leq|t| \leq 1$, by continuity, we get $L_{\infty}(s, \chi)$ is bounded. Hence we get (1).

(2) Now fix $H, \epsilon^{\prime}>0$. Let $s=-H+i T$, then have

$$
G_{1}=\prod_{v \in S} \frac{1-a_{v}^{-1} q_{v}^{-s}}{1-a_{v} q_{v}^{1-s}}
$$

where $a_{v}=\chi_{v}\left(\pi_{v}\right)$ while $\pi_{v}$ the uniformizer of $K_{v}$. Hence

$$
\begin{aligned}
\left|G_{1}\right| & <=\prod_{v \in S}\left(1+q_{v}^{H}\right)\left|L_{S}(1-s, \chi)\right| \\
& \leq N_{S}^{H} 2^{|S|} \zeta_{K, S}(1+H) \\
& <<_{H, n_{K}} N_{S}^{H} 2^{|S|}
\end{aligned}
$$

It suffices to show that $2^{|S|}<<_{H, n_{K}, \epsilon^{\prime}} N_{S}^{\epsilon^{\prime}}$. In fact, let $b=\left[2^{\epsilon^{\prime}}\right]+1$, then $2^{|S|}<2^{n_{K} b} N_{S}^{\epsilon^{\prime}}$ as when $\mathfrak{p}_{v}>b$ then $\mathfrak{p}_{v}^{\epsilon^{\prime}}>2$.

Done. 
Proof of Theorem 4.1. Cont. :

Now

$$
\begin{aligned}
S(X, \chi, S) & =\frac{1}{2 \pi i} \int_{(-H)} X^{s} W(s) W(\chi) A(\chi)^{1 / 2-s} G_{0}(s) G_{1}(s) L^{S}\left(1-s, \chi^{-1}\right) d s \\
& =\frac{1}{2 \pi i} \int_{(-H)} X^{s} W(s) W(\chi) A(\chi)^{1 / 2-s} G_{0}(s) G_{1}(s) \sum_{n=1}^{\infty} \frac{a_{\chi, S}(n)}{n^{1+H}} d s \\
& =\frac{1}{2 \pi i} \sum_{n=1}^{\infty} \frac{a_{\chi, S}(n)}{n^{1+H}} \int_{(-H)} X^{s} W(s) W(\chi) A(\chi)^{1 / 2-s} G_{0}(s) G_{1}(s) n^{s+H} d s
\end{aligned}
$$

Here the interchange of the sum and the integral is guaranteed by the absolute convergence of the Dirichlet series, rapid decay of $W(s)$. Applying Lemma 4.2, we have

$$
\begin{aligned}
& S(X, \chi, S)<<_{H, K} L^{S}(1+H, \chi) \int_{(-H)}\left|X^{s} W(s) W(\chi) A(\chi)^{1 / 2-s} G_{0}(s) G_{1}(s) d s\right| \\
& <<_{H, K} \zeta(1+H)^{n_{K}} X^{-H} N(\chi)^{1 / 2+H} N_{S}^{H} \int_{(-H)} W(s)(1+|t|)^{(1 / 2+H) n_{K}} d s \\
& \quad<<_{H, K} X^{-H} N(\chi)^{1 / 2+H} N_{S}^{H}
\end{aligned}
$$

To establish the theorem, we need to bound $S(X, \chi, S)$ below. Now assume that $\chi_{v}=1$ for all $v \notin S$ such that $N\left(\mathfrak{p}_{v}\right) \leq 3 X$. Then

$$
S(X, \chi, S)=S(X, 1, S)
$$

Note that $S(X, 1, S)$ is greater than $e^{-1}$ multiples of the primes $\mathfrak{p}_{v}$ of $K$ of degree 1 outside $S$ such that $X \leq \mathfrak{p}_{v} \leq 2 X$, By the prime number theorem, for some $A>0$ depending on $K$, when $X>A, S(X, 1, S)>X /(4 \log (X))-|S|$. Hence when $X>4 A|S|^{2}, S(X, 1, S)>X /(8 \log (X))$.

Then we have, when $X>4 A|S|^{2}$,

$$
X /(4 \log (X))<S(X, 1, S)=S(X, \chi, S)<C^{\prime} X^{-H} N(\chi)^{1 / 2+H} N_{S}^{H}
$$

for some $C^{\prime}$ depending on $H$ and $K$.

Therefore,

$$
\begin{aligned}
X^{H+1-\epsilon} & <<_{\epsilon} X^{H+1} \log (X)^{-1} \\
& <<_{H, K} N(\chi)^{1 / 2+H} N_{S}^{H}
\end{aligned}
$$

and thus

$$
X<<_{\epsilon, H, K} N(\chi)^{\frac{H+1 / 2}{H+1-\epsilon}} N_{S}^{\frac{H}{H+1-\epsilon}}
$$

Hence when $H$ is sufficiently small,

$$
X<C N(\chi)^{\epsilon+1 / 2} N_{S}^{\epsilon}
$$

where $C$ depends on $\epsilon$ and $K$. Thus, we have

$$
\begin{aligned}
X & <\operatorname{Max}\left(C N(\chi)^{\epsilon+1 / 2} N_{S}^{\epsilon}, 4 A|S|^{2}\right) \\
& <<_{\epsilon, K} N(\chi)^{\epsilon+1 / 2} N_{S}^{\epsilon}
\end{aligned}
$$


Hence Theorem 4.1 follows.

\section{2. $S$-version of Multiplicity One for $G L(n)$.}

In fact, such method can be applied to prove the following theorem. Here the analytic conductor is defined as in [6, [2, [13. See [27.

Theorem 4.4. Let $\pi=\otimes_{v} \pi_{v}$ and $\pi^{\prime}=\otimes_{v} \pi_{v}^{\prime}$ be two cuspidal automorphic representations of $\mathrm{GL}_{d}\left(\mathbb{A}_{K}\right)(d \geq 2)$, with analytic conductors $\leq Q$, and let $S$ be a finite set of places of $K$.

Then if Given an arbitrary $H>0$, then there exists a constant $C=C(H, d, K, \epsilon)$ such that, if $\pi_{v} \cong \pi_{v}^{\prime}$ for all places $v$ outside $S$ with the norm

$$
N\left(\mathfrak{p}_{v}\right) \leq C Q^{2 d+\epsilon} N_{S}^{d^{2}+\epsilon}
$$

Then $\pi \cong \pi^{\prime}$.

\section{REFERENCES}

[1] E. Artin and J. Tate, Class field theory, Harvard University 1968.

[2] F. Brumley, Effective multiplicity one on $\mathrm{GL}(N)$ and narrow zero-free regions for RankinSelberg L -functions, Amer. J. Math. 128 (2006), no. 6, 1455 C1474.

[3] J. Cassells and A. Froliche (editor), Algebraic Number Theory, Proceedings of the instructional conference held at the University of Sussex, Brighton, September 1C17, 1965. Academic Press, Inc (1967 reprinted).

[4] Friedlander, On the least $k$-th power non-residue in an algebraic number field, Proc. London Math. Soc. (3) 26 (1973), 19 C34.

[5] W. Grunwald, Ein Allgemeines Existenztheorem für algebraicshe Zahlkörper, Journ. f.d. reine u. angewante Math. 169, (1933) 103-107.

[6] J. Hoffstein and D. Ramakrishnan, Siegel Zeros and Cusp Forms, IMRN(1995), No.6, 279308.

[7] V.K. Murty, The least prime which does not split completely, (Communicated by Dinakar Ramakrishnan) Forum Math.6(1994), 555-565.

[8] E. Landau, Algebraische Zahlen, Göttingen 1927

[9] J.C. Lagarias, H.L. Montgomery and A.M. Odlyzko, A bound for the least prime ideal in the Chebotarev density theorem, Inventiones. Math. 54 271-196.

[10] J.C. Lagarias and A.M. Odlyzko, Effective Version of the Chebotarev Density Theorem Algebraic Number Fields, $L$-functions and Galois Properties (edit by A.Frölich) 409-464 (1977).

[11] S. Lang, Algebraic number theory, GTM 110, Springer-Verlag, 1970.

[12] S. Lang, Algebra, GTM 211, Springer,.

[13] Jianya Liu and Yonghui Wang, A theorem on analytic strong multiplicity one, J. Number Theory 129 (2009), no. 8, 1874C1882.

[14] Xiannan Li, The smallest prime that does not split completely in a number field, Algebra Number Theory 6 (2012), 1061-1096.

[15] C.J. Moreno, Analytic Proof of the strong multiplcity one, American Journal of Mathematics, Vol 107, no. 1, 163-206.

[16] J. Neukirch, Class field theory, Springer-Verlag, 1970.

[17] J. Neukirch, Algebraic number theory, Springer, 1991.

[18] A.M. Odlyzko, On conductors and discriminants, Algebraic Number Fields, $L$-functions and Galois Properties (edit by A.Frölich) 377-407 (1977).

[19] D. Ramakrishnan and R.J. Valenza, Fourier Analysis on Number Fields Springer, 1999. 
[20] D. Ramakrishnan and Song Wang, On the exceptional zeros of Rankin-Selberg L-functions, Compositio Math. 135 (2003), no. 2, 211C244.

[21] J.-P. Serre, Quelques applications du théorème de densité de Chebotarev, Publ.Math. I.H.E.S., n 54 (1981) 123-201

[22] J.-P. Serre, E UVRES Collected Papers, Vol III, Springer Verlag. No.125. (563-641).

[23] H. Stark, Some effective cases of the Brauer-Siegel Theorem, Inventiones Math. (23) 1974, $135-162$.

[24] J. Voloch, Chebyshev's method for number fields, J. Thor. Nombres Bordeaux 12 (2000), no. 1, $81 \mathrm{C} 85$.

[25] J.D. Vaaler and J. Voloch, The Least Nonsplit Prime in Galois extensions of $\mathbb{Q}$, J. Number Theory 85 (2000), no. 2, 320C335.

[26] Song Wang, An effective version of the Grunwald-Wang Theorem, Ph'D Thesis (2001, Caltech).

[27] Song Wang, Multiplicity One, S-version, preprint.

[28] Yonghui Wang, The analytic strong multiplicity one theorem for $\mathrm{GL}_{m}\left(\mathbb{A}_{K}\right)$. J. Number Theory 128 (2008), no. 5, 1116C1126.

[29] Sh. Wang, A counter example to Grunwald's theorem, Annals of Math. 49 (1948) 1008-1009.

[30] Sh. Wang, On Grunwald's theorem, Annals of Math. 51 (1950) 471-484.

[31] G. Whaples, No-analytic class field theory and Gruenwald's Theorem, Duke Math. J. 9 (1942) 455-473

[32] A. Weil, Sur les "formules explicites" de la théorie des nombres premiers, Comm. Sem. Math. Lund, 252-265 (1952).

[33] A. Weil, Basic Number Theory, 1974.

Song Wang, Academy of Mathematics and Systematics Sciences, the Morningside Center of Mathematics, the Key Laboratory of Hua, Chinese Academy of Science.

E-mail address: songw1973@amss.ac.cn 